\title{
The structure of generic anomalous dimensions and no- $\pi$ theorem for massless propagators
}

\author{
P.A. Baikov ${ }^{a}$ and K.G. Chetyrkin ${ }^{b}$ \\ a Skobeltsyn Institute of Nuclear Physics, Lomonosov Moscow State University, \\ 1(2), Leninskie gory, Moscow 119991, Russian Federation \\ ${ }^{b}$ II Institut für Theoretische Physik, Universität Hamburg, \\ Luruper Chaussee 149, 22761 Hamburg, Germany \\ E-mail: baikov@theory.sinp.msu.ru, Konstantin.Chetyrkin@desy.de
}

ABSTRACT: Extending an argument of [1] for the case of 5-loop massless propagators we prove a host of new exact model-independent relations between contributions proportional to odd and even zetas in generic $\overline{\mathrm{MS}}$ anomalous dimensions as well as in generic massless correlators. In particular, we find a new remarkable connection between coefficients in front of $\zeta_{3}$ and $\zeta_{4}$ in the 4-loop and 5-loop contributions to the QCD $\beta$-function respectively. It leads to a natural explanation of a simple mechanics behind mysterious cancellations of the $\pi$-dependent terms in one-scale Renormalization Group (RG) invariant Euclidean quantities recently discovered in [2]. We give a proof of this no- $\pi$ theorem for a general case of (not necessarily scheme-independent) one-scale massless correlators. All $\pi$-dependent terms in the six-loop coefficient of an anomalous dimension (or a $\beta$-function) are shown to be explicitly expressible in terms of lower order coefficients for a general one-charge theory. For the case of a scalar $O(n) \phi^{4}$ theory all our predictions for $\pi$-dependent terms in 6-loop anomalous dimensions are in full agreement with recent results of [3-5].

KEYwords: Perturbative QCD, Renormalization Group

ARXIV EPRINT: 1804.10088 


\section{Contents}

1 Introduction 1

2 Setup 3

3 Interplay between $\zeta_{3}, \zeta_{4}:$ generic case $\quad 7$

4 Interplay between $\zeta_{5}, \zeta_{6}:$ QCD case 9

5 The connection between $\zeta_{3}$ and $\zeta_{4}$ terms in the QCD $\beta$-function 10

6 The $\pi-\zeta-\epsilon$ structure of p-integrals and renormalization constants 11

$7 \pi$-free representation for 5- and 6-loop p-integrals 13

8 Adler function at order $\alpha_{s}^{5} \quad 15$

9 All order no- $\pi$ theorem $\quad 15$

10 Odd and even zetas in RG-functions up to 6 loops 16

11 Odd and even zetas in p-functions up to 5 loops 20

$\begin{array}{ll}12 \text { Discussion and conclusions } & 21\end{array}$

\section{Introduction}

The seminal calculation of the Adler function at order $\alpha_{s}^{3}[6]$ demonstrated for the first time a mysterious complete cancellation of all contributions proportional to $\zeta_{4}$ (abounding in separate diagrams) while odd zetas terms (that is those proportional to $\zeta_{3}$ and $\zeta_{5}$ ) survived and appeared in the final result. Literally the authors of [6] wrote: "We would like to stress the cancellations of $\zeta_{4}$ in the final results for $R(s)$. It is very interesting to find the origin of the cancellation of $\zeta_{4}$ in the physical quantity."

Since then it has been noted many times that all one scale physical quantities are indeed free from even zetas at order $\alpha_{s}^{4}$ (like corrections to the Bjorken (polarized) DIS sum rule) and some of them - like the Adler function - even at next, in fact, five-loop, $\alpha_{e} \alpha_{s}^{4}$ order [7]. By one-scale physical quantities we mean here scale invariant massless correlators (or their proper combinations) in the Euclidean region. ${ }^{1}$

\footnotetext{
${ }^{1}$ We do not consider well known terms proportional to variouus powers of $\pi$ which are routinely generated during the procedure of analytical continuation to the Minkowskian (negative) values of the momentum transfer $Q^{2}$.
} 
The appearance of $\zeta_{4}$ in a one-scale physical quantity has been demonstrated in [8] for the case of the 5-loop scalar correlator.

Very recently M. Jamin and R. Miravitllas have discovered that after a transition to a new so-called C-scheme [9] all terms proportional to even zetas $\left(\zeta_{4}\right.$ and $\zeta_{6}$ in the cases under consideration) do disappear in the 5-loop scalar correlator as well as in the 5-loop gluon correlator [2] (both enter the hadronic decays of the Higgs boson [10, 11]). They also suggested that the absence of even zetas after transition to the C-scheme is an universal feature of all $\mathcal{O}\left(\alpha_{s}^{5}\right)$ physical quantities ${ }^{2}$ — "no- $\pi^{2}$ conjecture".

Later many more confirmations of the conjecture have been discussed in [12-14].

The most interesting feature of the conjecture is a direct universal connection between $\zeta_{4}$ contribution to physical quantities at order $\alpha_{s}^{5}$ and the $\zeta_{4}$-term in the QCD $\beta$-function which appears first at order $\alpha_{s}^{5}[15-17]$. To really appreciate the mystery behind these cancellations we want to remind the reader of the following simple facts:

1. a bare physical (massless!) quantity depends on the bare coupling constant, $\alpha_{s}^{B}$;

2. its renormalization is done with the replacement $\alpha_{s}^{B}=\mu^{2 \epsilon} Z_{a} \alpha_{s}$;

3. the charge Renormalization Constant (RC) $Z_{a}$ depends on the five-loop coefficient in the $\beta$-function $-\beta_{5}$ - starting from the fifth order, $\alpha_{s}^{5}$;

4. as a result the renormalized physical quantity starts to "feel" $\beta_{5}$ only at the astonishingly large sixth order in $\alpha_{s}$;

5. for the case of the scalar correlator the contribution of order $\alpha_{s}^{6}$ corresponds to the fabulously large 7-loop level.

In the present work we suggest a natural explanation of the mystery above as well as a rigorous proof of the no- $\pi^{2}$ conjecture (at least for the 5-loop level), by demonstrating that the $\zeta_{4}$ term in the $\beta$-function is, in fact, not independent but must meet a simple factorization formula:

$$
\beta_{5}^{\zeta_{4}}=\frac{9}{8} \beta_{1} \beta_{4}^{\zeta_{3}},
$$

where the $\beta$-function is defined as:

$$
\mu^{2} \frac{\mathrm{d}}{\mathrm{d} \mu^{2}} a(\mu)=a \beta(a)=a \sum_{i \geq 1} \beta_{i} a^{i}, \quad a=\frac{\alpha_{s}}{4 \pi}=\frac{g_{s}^{2}}{16 \pi^{2}}
$$

and the upper-script $\zeta_{i}$ means

$$
F^{\zeta_{i}}=\lim _{\zeta_{i} \rightarrow 0} \frac{\partial}{\partial \zeta_{i}} F \text { and (for future reference) } F^{\zeta_{i} \zeta_{j}}=\lim _{\zeta_{i} \rightarrow 0} \frac{\partial}{\partial \zeta_{i}} F^{\zeta_{j}} .
$$

The validity of the factorization formula will be proven without any loop calculation and (almost) without any information on the $\beta$-function. The proof is based solely on the

\footnotetext{
${ }^{2}$ Please, pay attention that the counting of powers of $\alpha_{s}$ is definition depended, see more details in the next section.
} 
general considerations about the structure of the 4-loop massless propagators discussed in section 7 of [1] and the fact that the 3-loop QCD $\beta$-function is free from any zetas $[18,19]$.

Note that factorization in (1.1) is not trivial even for QCD with the SU(3) gauge group (the coefficient $\beta_{4}$ was first computed in [20] and confirmed in [21]):

$$
\begin{gathered}
\beta_{1} \\
\frac{\partial}{\partial \zeta_{4}} \beta_{5}=\frac{9}{8}\left(\frac{2}{3} n_{f}-11\right)\left(-\frac{6472}{81} n_{f}^{2}+\frac{6508}{27} n_{f}-3564\right)
\end{gathered}
$$

while for a general case it takes the form:

$$
\begin{aligned}
\beta_{1} & \left(\partial / \partial \zeta_{3}\right) \beta_{4} \\
\frac{\partial}{\partial \zeta_{4}} \beta_{5}= & \frac{9}{8}\left(\frac{4}{3} n_{f} T_{F}-\frac{11}{3} C_{A}\right)\left(\frac{44}{9} C_{A}^{4}-\frac{136}{3} C_{A}^{3} n_{f} T_{F}\right. \\
& +\frac{656}{9} C_{A}^{2} C_{F} n_{f} T_{F}-\frac{224}{9} C_{A}^{2} n_{f}^{2} T_{F}^{2}-\frac{352}{9} C_{A} C_{F}^{2} n_{f} T_{F} \\
& -\frac{448}{9} C_{A} C_{F} n_{f}^{2} T_{F}^{2}+\frac{704}{9} C_{F}^{2} n_{f}^{2} T_{F}^{2}-\frac{704}{3} \frac{d_{A}^{a b c d} d_{A}^{a b c d}}{N_{A}} \\
& \left.+\frac{1664}{3} \frac{d_{F}^{a b c d} d_{A}^{a b c d}}{N_{A}} n_{f}-\frac{512}{3} \frac{d_{F}^{a b c d} d_{F}^{a b c d}}{N_{A}} n_{f}^{2}\right) .
\end{aligned}
$$

We will derive a variety of exact relations similar to (1.1) valid for arbitrary $\overline{\mathrm{MS}}[22,23]$ anomalous dimensions (ADs). We also will find new identities which relate contributions proportional to even zetas in (not necessarily physical) massless correlators and terms proportional to odd zetas in the corresponding ADs.

In addition we suggest a simple proof of the no- $\pi$ theorem valid for any number of loops and every massless correlator (including those with non-zero AD) provided the participating Feynman integrals meet a simple condition to be specified later. The condition is shown to be fulfilled for all currently known examples of the theorem. We also construct explicit expressions for the $\pi$-dependent contributions to 6-loop ADs and $\beta$-function valid for a generic one-charge theory.

If not otherwise stated we will assume the so-called $G$-scheme for renormalization [24]. The scheme is natural for massless propagators. All ADs, $\beta$-functions and $Z$-factors are identical in $\overline{\mathrm{MS}}$ - and G-schemes. For (finite) renormalized functions there exists a simple conversion rule. Namely, in order to switch from an $G$-renormalized quantity to the one in the $\overline{\mathrm{MS}}$-scheme one should make the following replacement in the former: $\ln \mu^{2} \rightarrow \ln \mu^{2}+2$ ( $\mu$ is the renormalization scale, the limit of $\epsilon \rightarrow 0$ is understood).

\section{Setup}

Let $F$ be any $p$-function, that is a Green function or a 2-point correlator (or even some combination thereof), expressible in terms of massless propagator-like Feynman integrals (to be named p-integrals below). As an example one could have in mind any of the 11 
objects computed and renormalized at the 4-loop level in [25]. $F$ may not necessarily be a gauge invariant object, if there is a gauge dependence we will always assume the Landau gauge fixing condition below. With such convention at hand we can and will effectively ignore the gauge parameter dependence (if any) in all objects under consideration here. The (renormalized) p-function $F \equiv F_{R}$ can be naturally represented as

$$
F_{n}\left(a, \ell_{\mu}\right)=1+\sum_{1 \leq i \leq n}^{0 \leq j \leq i} g_{i, j} \ell_{\mu}^{j} a^{i},
$$

where $a=\frac{\alpha_{s}(\mu)}{4 \pi}, \ell_{\mu}=\ln \frac{\mu^{2}}{Q^{2}}$ and $Q$ is an (Euclidean) external momentum. The integer $n$ stands for the (maximal) power of $\alpha_{s}$ appearing in the Feynman diagrams contributing to $F_{n}$. The $F$ without $n$ will stand as a shortcut for a formal series $F_{\infty}$. In terms of bare quantities $F$ is written as

$$
F=Z F_{B}\left(a_{B}, \ell_{\mu}\right), \quad Z=1+\sum_{i \geq 1}^{1 \leq j \leq i} Z_{i, j} \frac{a^{i}}{\epsilon^{j}},
$$

with the bare coupling constant and the corresponding RC being

$$
a_{B}=\mu^{2 \epsilon} Z_{a} a, \quad Z_{a}=1+\sum_{i \geq 1}^{1 \leq j \leq i}\left(Z_{a}\right)_{i, j} \frac{a^{i}}{\epsilon^{j}} .
$$

The evolution equation for $\mathrm{F}$ reads:

$$
\left(\frac{\partial}{\partial \ell_{\mu}}+\beta a \frac{\partial}{\partial a}\right) F=\gamma F
$$

with the AD

$$
\gamma(a)=\sum_{i \geq 1} \gamma_{i} a^{i}, \quad \gamma_{i}=-i Z_{i, 1}
$$

The coefficients of the $\beta$-function $\beta_{i}$ are related to $Z_{a}$ in the standard way:

$$
\beta_{i}=i\left(Z_{a}\right)_{i, 1} .
$$

Note that if the $\mathrm{AD} \gamma$ happens to be equal $\beta$ for a p-function $F$ (an explicit example will be considered later) then the latter should be renormalized with $Z \equiv\left(Z_{a}\right)^{-1}$ (due to different signs in the above expressions for $\gamma_{i}$ and $\beta_{i}$ ).

Currently our ability to compute p-functions in QCD is limited to 4 loops and the corresponding ADs to 5 loops (see, e.g. [26]). If $\gamma$ is not vanishing then there are two ways to construct a scale-invariant version of $F$.

Let us consider them in turn. The first one is to construct an object:

$$
\begin{aligned}
\hat{F}^{\mathrm{s} i}\left(a, \ell_{\mu}\right)_{n+1} & =(a)^{\frac{-\gamma_{1}}{\beta_{1}}} \exp \left\{-\int_{0}^{a} \frac{\mathrm{d} x}{x}\left[\frac{\gamma(x)}{\beta(x)}-\frac{\gamma_{1}}{\beta_{1}}\right]\right\} F_{n}\left(a, \ell_{\mu}\right) \\
& =(a)^{\frac{-\gamma_{1}}{\beta_{1}}}\left(1+\sum_{1 \leq i \leq n}^{0 \leq j \leq i} \hat{d}_{i, j} a^{i} \ell_{\mu}^{j}\right)
\end{aligned}
$$

where $a=a(\mu)$. Note that $\hat{F}_{n+1}^{\mathrm{si}}$ is built from $F_{n}$ and the $(\mathrm{n}+1)$-loop $\mathrm{AD} \gamma$. 
We will be interested in even zetas appearing in the coefficients $\hat{d}_{i} \equiv \hat{d}_{i 0}$. It is a wellknow fact (see discussion in section 6) that even zeta may appear only starting from 3 loops for correlators and from 4 loops for ADs. In addition $\beta_{4}$ does not depend on $\zeta_{4}$ (this is a peculiar property of $\beta_{4}$ in QCD). An account of this directly leads to the following representations for the (potentially) $\pi$-dependent contributions to $\hat{d}_{3}$ and $\hat{d}_{4}$ :

$$
\begin{aligned}
& \hat{d}_{3} \stackrel{\pi}{=}-\frac{1}{3 \beta_{1}}\left(\gamma_{4}-3 \beta_{1} g_{3}\right), \\
& \hat{d}_{4} \stackrel{\pi}{=}-\frac{1}{4 \beta_{1}}\left(\gamma_{5}-\frac{\gamma_{1} \beta_{5}}{\beta_{1}}-\frac{\gamma_{4} \beta_{2}}{\beta_{1}}+\frac{4 \gamma_{4} g_{1}}{3}-4 \beta_{1} g_{4}\right),
\end{aligned}
$$

where the sign $\stackrel{\pi}{=}$ means equality modulo $\pi$-independent terms. Note that in eq. (2.10) we have used the fact (proven in [1] and discussed later ${ }^{3}$ ) that $\hat{d}_{3} \stackrel{\pi}{=} 0$.

Unlike the 3-loop level, the 4-loop coefficient $\hat{d}_{4}$ does provide no-zero contribution proportional to $\zeta_{4}$ in every available example with $\gamma \neq 0$, but terms with $\zeta_{6}$ and $\zeta_{3} \zeta_{4}$ never show up. It is of interest that the second combination which does appear in many master integrals $[1,27]$ never contributes to all known 4-loop renormalized correlators (not necessarily scale-invariant) and 5-loop ADs. The reason for it will be clarified in section 6 .

The remarkable observation first made in [2] can, in our setup, be precisely formulated as follows:

$$
\hat{d}_{4} \stackrel{\pi}{=} \frac{\gamma_{1} \beta_{5}}{3 \beta_{1}^{2}}
$$

or, in an explicit form,

$$
\hat{d}_{4}-\frac{\gamma_{1} \beta_{5}}{3 \beta_{1}^{2}} \stackrel{\pi}{=}-\frac{1}{4 \beta_{1}}\left(\frac{\gamma_{1} \beta_{5}}{3 \beta_{1}}+\gamma_{5}-\frac{\gamma_{4} \beta_{2}}{\beta_{1}}+\frac{4 \gamma_{4} g_{1}}{3}-4 \beta_{1} g_{4}\right) \stackrel{\pi}{=} 0 .
$$

We will prove (2.12) in section 3.

If we change the renormalization scheme as follows:

$$
a=\bar{a}\left(1+c_{1} \bar{a}+c_{2} \bar{a}^{2}+c_{3} \bar{a}^{3}+c_{4} \bar{a}^{4}\right)
$$

with $c_{1}, c_{2}$ and $c_{3}$ all free from even zetas and with

$$
c_{4} \stackrel{\pi}{=} \frac{1}{3} \frac{\beta_{5}}{\beta_{1}} \stackrel{\pi}{=} \frac{3}{8} \beta_{4}^{\zeta_{3}} \zeta_{4}
$$

then the function $\hat{F}_{5}^{\mathrm{si}}\left(\bar{a}, \ell_{\mu}\right)$ as well the (5-loop) $\beta$-function $\bar{\beta}(\bar{a})$ both loose any dependence on even zetas. We will call the class of renormalization schemes for which

$$
\bar{\beta}(\bar{a}) \stackrel{\pi}{=} \mathcal{O}\left(\bar{a}^{L+1}\right) \text { and } c_{1} \stackrel{\pi}{=} 0
$$

as $\pi$-independent to $\mathrm{L}$ loops schemes. Note that any two $\pi$-independent (to L loops) schemes are related by transformation (2.13) with all coefficients $c_{1} \ldots c_{L-1}$ being $\pi$-free.

\footnotetext{
${ }^{3}$ See eq. (3.3).
} 
An example of such a scheme has been recently considered in [9] under the name "C-scheme". In this scheme the $\beta$-function is $\pi$-independent in all orders. In particular,

$$
c_{4}=\frac{1}{3} \frac{\beta_{5}}{\beta_{1}} .
$$

The second way to construct a scale-invariant object from $F$ is also well-known: ${ }^{4}$

$$
F_{n+1}^{\mathrm{si}}\left(a, \ell_{\mu}\right)=\frac{\partial}{\partial \ell_{\mu}}(\ln F)_{n+1} \equiv\left(\frac{\left(\gamma(a)-\beta(a) a \frac{\partial}{\partial a}\right) F_{n}}{F_{n}}\right)_{n+1} .
$$

Note that $F_{n+1}^{\mathrm{s} i}\left(a, \ell_{\mu}\right)$ starts from the first power of the coupling constant $a$ and is formally composed from $\mathcal{O}\left(\alpha_{s}^{n+1}\right)$ Feynman diagrams. In the same time is can be completely restored from $F_{n}$ and the $(n+1)$-loop $\mathrm{AD} \gamma$. This dual nature of $F_{n+1}^{\mathrm{si}}$ plays a vital role in all our considerations in the next two sections.

It is convenient to write

$$
F_{n+1}^{\mathrm{si}}\left(a, \ell_{\mu}\right)=a\left(\gamma_{1}+\sum_{1 \leq i \leq n}^{0 \leq j \leq i} d_{i, j} \ell_{\mu}^{j} a^{i}\right) .
$$

The coefficients $d_{i}=d_{i 0}$ meet, obviously, the equations (parallel to (2.9) and (2.10)):

$$
\begin{aligned}
& d_{3} \stackrel{\pi}{=} \gamma_{4}-3 \beta_{1} g_{3}, \\
& d_{4} \stackrel{\pi}{=} \gamma_{5}-\frac{\gamma_{4} \beta_{2}}{\beta_{1}}+\frac{4 \gamma_{4} g_{1}}{3}-4 \beta_{1} g_{4} .
\end{aligned}
$$

Note, that up to a common factor $\hat{d}_{3}$ is equal to $d_{3}$; the same is true for the pair $\hat{d}_{4}$ and $d_{4}$ except for a term proportional to $\beta_{5}$ which does not appear at all in $d_{n}$ if $n \leq 4$. A transition to a $\pi$-independent renormalization scheme according to (2.13) and (2.14) leads to

$$
F_{5}^{\mathrm{si}}\left(\bar{a}, \ell_{\mu}\right)=a\left(\gamma_{1}+\sum_{1 \leq i \leq 4}^{0 \leq j \leq i} \bar{d}_{i, j} \ell_{\mu}^{j} \bar{a}^{i}\right)
$$

with

$$
\bar{d}_{2} \stackrel{\pi}{=} d_{2}, \bar{d}_{3} \stackrel{\pi}{=} d_{3}, \bar{d}_{4} \stackrel{\pi}{=} d_{4}+\frac{\gamma_{1} \beta_{5}}{3 \beta_{1}} \stackrel{\pi}{=} \frac{\gamma_{1} \beta_{5}}{3 \beta_{1}}+\gamma_{5}-\frac{\gamma_{4} \beta_{2}}{\beta_{1}}+\frac{4 \gamma_{4} g_{1}}{3}-4 \beta_{1} g_{4} .
$$

Eqs. (2.12) and (2.22) explicitly demonstrate that if, for a given p-function $F_{4}$, its scale invariant version $F_{5}^{\mathrm{si}}\left(a, \ell_{\mu}\right)$ is $\pi$-free in a $\pi$-independent renormalization scheme then the function $\hat{F}_{5}^{\mathrm{si}}\left(a, \ell_{\mu}\right)$ is also $\pi$-free, and vice versa.

Our main aim is to really understand and, consequently, to prove the equality (which happens to be true in all known so far examples)

$$
d_{4}+\frac{1}{3} \frac{\gamma_{1} \beta_{5}}{\beta_{1}} \stackrel{\pi}{=} 0
$$

\footnotetext{
${ }^{4}$ The trick was first applied for constructing a $\pi$-free version of a 2-point correlator in [28]. The same quantity as defined in (2.17) was discussed in [29] under the name of the "Renormalization Scheme invaraint anomalous dimension". In DIS similar objects are called "physical anomalous dimensions", see, e.g. [12].
} 
The equality is naturally separated into two pieces

$$
\begin{array}{r}
d_{4}+\frac{1}{3} \frac{\gamma_{1} \beta_{5}^{\zeta_{4}}}{\beta_{1}} \zeta_{4} \stackrel{\pi^{4}}{=} 0, \\
d_{4} \stackrel{\pi^{6}}{=} 0 .
\end{array}
$$

Assuming the factorization formula (1.1) (which will be derived in section 5), we will prove in what follows that

$$
\begin{array}{r}
d_{4}+\frac{3}{8} \gamma_{1} \beta_{4}^{\zeta_{3}} \zeta_{4} \stackrel{\pi^{4}}{=} 0 \\
d_{4} \stackrel{\pi^{6}}{=} 0 .
\end{array}
$$

\section{$3 \quad$ Interplay between $\zeta_{3}, \zeta_{4}$ : generic case}

In general representation (2.17) is very suitable to discuss the interplay of various transcendental contributions to $F, \beta$ and $\gamma$. This is because, unlike $(2.8), F_{n}^{\mathrm{si}}\left(a, \ell_{\mu}\right)$ is a combination of a finite number of Feynman diagrams with a very simple renormalization mode: $F^{\mathrm{s} i}\left(a, \ell_{\mu}\right)=F_{B}^{\mathrm{si}}\left(a_{B}=Z_{a} \mu^{2 \epsilon} a, \ell_{\mu}\right)$ (as we discussed already in the Introduction).

The (bare) function $F_{5, B}^{\mathrm{si}}$ is composed from 1,2,3,4 and 5-loop bare p-integrals. ${ }^{5}$ We will call a (bare) $L$-loop p-integral $F\left(Q^{2}, \epsilon\right) \pi$-safe if the $\pi$-dependence of its pole in $\epsilon$ and constant part can be completely absorbed into the properly defined "hatted" odd zetas. The first observation of a non-trivial class of $\pi$-safe p-integrals - all 3-loop ones - was made in [30]. An extension of the observation on the class of all 4-loop p-integrals was performed in [1]. Here it was shown that, given an arbitrary 4-loop p-integral, its pole in $\epsilon$ and constant part depend on even zetas only via the following combinations:

$$
\hat{\zeta}_{3}:=\zeta_{3}+\frac{3 \epsilon}{2} \zeta_{4}-\frac{5 \epsilon^{3}}{2} \zeta_{6}, \hat{\zeta}_{5}:=\zeta_{5}+\frac{5 \epsilon}{2} \zeta_{6} \quad \text { and } \quad \hat{\zeta}_{7}:=\zeta_{7}
$$

Note that the $\pi$-safeness of, say, all $L$-loop p-integrals ensures that for any $L^{\prime}$-loop $p$-integral $F$ with $L^{\prime}<L$ the $L$-loop combination $\frac{1}{\epsilon^{L-L^{\prime}}} F$ is a $\pi$-safe one with the same choice of the hatted zetas.

We will proceed, assuming for the moment that all p-integrals contributing to $F_{5}^{\text {si }}$ are $\pi$-safe. The assumption is discussed and eventually proved in section 7 (at least for the particular classes of p-integrals which we encounter in the present paper).

Any renormalized p-function $F_{5, R}$ can, by definition, be presented as

$$
F_{5, R}=F_{5, B}+\sum_{i} Z_{i} p_{i}
$$

where $Z_{i}$ are some UV counterterms and $p_{i}$ are p-integrals. Let us assume for a moment that all $Z_{i}$ in (3.2) are $\pi$-free and rewrite all (including those contributing to $F_{n}^{B}$ ) p-integrals

\footnotetext{
${ }^{5}$ In some cases (correlators of composite operators) also 6-loop p-integrals might contribute (see a discussion in section 7).
} 
in (3.2) in terms of hatted zetas. Then the r.h.s. of (3.2) will depend on hatted odd zetas only (in the limit of $\epsilon \rightarrow 0$ ). The finiteness of $F_{5, R}$ would ensure then the absence of any $\pi$ dependent terms in it in the $\epsilon \rightarrow 0$ limit. Clearly, if some of $Z_{i}$ do contain odd and/or even zetas then there should exist some constraints relating contributions with odd and even zetas to the p-function and depending on a precise pattern of hiding $\pi$-dependent terms inside hatted odd zetas. These constraints will be the main tool in our considerations.

Let us consider first $F_{4}^{\mathrm{si}}$. It is renormalized with $Z_{a}$ in three loops which is free from any zetas for QCD. As a result, $F_{4}^{\text {si }}$ should be free from even zetas which means the fulfillment of the identity

$$
d_{3} \stackrel{\pi}{=} \gamma_{4}-3 \beta_{1} g_{3} \stackrel{\pi}{=} 0 .
$$

Now let us turn to the renormalization of the very function $F_{3}$. Its renormalization mode includes $Z_{a}$ (in 2 loops, free from any zetas) and $Z_{\gamma}$ (in 3 loops). Note that $\left(Z_{\gamma}\right)_{3}$ depends generically on the combination $\zeta_{3} / \epsilon$. By definition of the AD

$$
\left(Z_{\gamma}\right)_{3,1}^{\zeta_{3}}=-\frac{1}{3} \gamma_{3}^{\zeta_{3}}
$$

Let us write the renormalized function $F_{3}$ as follows ${ }^{6}$

$$
F_{3}=\left(Z_{\gamma}\right)_{3,1}^{\zeta_{3}} \zeta_{3} \frac{a^{3}}{\epsilon}+\sum_{i} z_{i}(\epsilon) p_{i} a^{3}+\text { terms of order } a^{2} \text { and lower }
$$

where $p_{i}$ are some bare p-integrals and the coefficients $z_{i}$ are some polynomials in $1 / \epsilon$ (the constant term is allowed!) with rational coefficients. Assume now that the p-integrals in the above sum are expressed via hatted zetas. The coefficient in front of $\hat{\zeta}_{3}$ should then be equal

$$
-\left(Z_{\gamma}\right)_{3,1}^{\zeta_{3}} \frac{a^{3}}{\epsilon} \equiv \gamma_{3}^{\zeta_{3}} \frac{a^{3}}{3 \epsilon}
$$

Expressing back $\hat{\zeta_{3}}$ via normal zetas we arrive at the identity:

$$
g_{3}^{\zeta_{4}} \equiv \frac{1}{2} \gamma_{3}^{\zeta_{3}}
$$

Now a look on (3.3) immediately leads to relations valid for any $\mathrm{AD} \gamma$

$$
\gamma_{4}^{\zeta_{4}}=\frac{3}{2} \beta_{1} \gamma_{3}^{\zeta_{3}} .
$$

The fact that $\beta_{3} \stackrel{\pi}{=} 0$ is a particular feature of QCD. The above treatment can be easily extended for a general case of $\beta_{3}^{\zeta_{3}} \neq 0$. The corresponding generalizations of eqs. (3.3) and (3.7) read

$$
\begin{aligned}
d_{3} \stackrel{\pi}{=} \gamma_{4}-3 \beta_{1} g_{3} \stackrel{\pi}{=}-\frac{1}{2} \beta_{3}^{\zeta_{3}} \gamma_{1} \zeta_{4}, \\
\gamma_{4}^{\zeta_{4}}=\frac{3}{2} \beta_{1} \gamma_{3}^{\zeta_{3}}-\frac{1}{2} \beta_{3}^{\zeta_{3}} \gamma_{1} .
\end{aligned}
$$

For a particular case of $\gamma \equiv \beta$ we arrive at

$$
\beta_{4}^{\zeta_{4}}=\beta_{1} \beta_{3}^{\zeta_{3}} .
$$

\footnotetext{
${ }^{6}$ For simplicity we set $\mu=1$ in all formulas below.
} 


\section{Interplay between $\zeta_{5}, \zeta_{6}:$ QCD case}

Let us now add one more loop and consider $F_{5}^{\mathrm{si}}$. It is renormalized with the $\mathrm{RC} Z_{a}$ (taken in the 4-loop approximation):

$$
F_{5}^{\mathrm{si}}=\gamma_{1}\left(Z_{a}\right)_{4,1}^{\zeta_{3}}\left(\hat{\zeta}_{3}-\frac{3 \epsilon}{2} \zeta_{4}\right) \frac{a^{5}}{\epsilon}+\sum_{i, 1 \leq j \leq 5} z_{i, j}(\epsilon) \hat{p}_{i, j} a^{j}+\mathcal{O}\left(\epsilon^{0}\right),
$$

where $\hat{p}_{i, j}$ stands for $p$-integrals expressed in terms of hatted (odd!) zetas and $z_{i, j}(\epsilon)$ are completely rational polynomials in $1 / \epsilon$. The finiteness of (4.1) means that the term proportional to $\hat{\zeta}_{5}$ in the underlined sum in (4.1) should have a finite coefficient. Thus, the whole p-function $F_{5}^{\mathrm{si}}\left(a, \ell_{\mu}\right)$ should be free from $\zeta_{6}$ in the limit $\epsilon \rightarrow 0$. This could be precisely written as $d_{4} \stackrel{\pi^{6}}{=} 0$ or, equivalently,

$$
\gamma_{5}-\frac{\gamma_{4} \beta_{2}}{\beta_{1}}+\frac{4 \gamma_{4} g_{1}}{3}-4 \beta_{1} g_{4} \stackrel{\pi^{6}}{=} \gamma_{5}-4 \beta_{1} g_{4} \stackrel{\pi^{6}}{=} 0,
$$

where we have used the fact that $\gamma_{4} \stackrel{\pi^{6}}{=} 0$ (see table 1 ). On the other hand, the pole part of the coefficient in front of $\hat{\zeta}_{3}$ in the underlined sum must be equal to $-\gamma_{1}\left(Z_{a}\right)_{4,1}^{\zeta_{3}} / \epsilon$. This directly leads to the identity

$$
d_{4} \stackrel{\pi}{=}-\gamma_{1} \frac{3}{8} \beta_{4}^{\zeta_{3}} \zeta_{4}
$$

Thus, we have proved the no- $\pi$ theorem: for every (QCD!) p-function $F_{4}$ the corresponding scheme-invariant function $F_{5}^{\mathrm{si}}$ is $\pi$-independent. We will rederive it for generic functions $F_{n+1}^{\text {si }}$ it more systematic way in section 9 .

Let us write now the renormalized version of $F_{4}$ in a form explicitly showing all possible zetas coming from RCs. The form reads (an upper index $n \ell$ in $\hat{p}_{i}^{n \ell}$ means that the p-integral $p_{i}$ has $n$ loops)

$$
F_{4} \stackrel{\pi^{6}}{=} a^{4}\left[Z_{3,1}^{\zeta_{3}} \frac{\zeta_{3}}{\epsilon} \sum_{i} \hat{p}_{i}^{1 \ell}+Z_{4,2}^{\zeta_{3}} \frac{\zeta_{3}}{\epsilon^{2}}+Z_{4,1}^{\zeta_{3}} \frac{\zeta_{3}}{\epsilon}+Z_{4,1}^{\zeta_{4}} \frac{\zeta_{4}}{\epsilon}+Z_{4,1}^{\zeta_{5}} \frac{\zeta_{5}}{\epsilon}+\sum_{i} \hat{p}_{i}^{4 \ell}\right],
$$

where we have discarded all terms obviously independent of $\zeta_{6}$. The finiteness of coefficient in front of $\zeta_{5}$ in (4.4) leads to the equality

$$
\left(\sum_{i} \hat{p}_{i}^{4 \ell}\right)^{\hat{\zeta}_{5}}=-Z_{4,1}^{\zeta_{5}} / \epsilon
$$

Thus,

and, finally, after the use of (4.2)

$$
g_{4} \stackrel{\pi^{6}}{=}-\frac{5}{2} Z_{4,1}^{\zeta_{5}} \zeta_{6} \stackrel{\pi^{6}}{=} \frac{5}{8} \gamma_{4}^{\zeta_{5}} \zeta_{6}
$$

$$
\gamma_{5}^{\zeta_{6}}=\frac{5}{2} \gamma_{4}^{\zeta_{5}} \beta_{1}
$$

All derivations of this section have been done for the QCD case, that is assuming $\beta_{3}^{\zeta_{3}}=0$ and $\beta_{4}^{\zeta_{5}}=0$. A generalization to a generic one-charge theory can be also done along the same lines. But we postpone this to sections 9, 10 and 11 where we will suggest an universal treatment. 


\section{The connection between $\zeta_{3}$ and $\zeta_{4}$ terms in the QCD $\beta$-function}

As is well-known the use of the Landau gauge implies that

$$
Z_{a}=\left(Z^{\mathrm{gh}}\right)^{-2}\left(Z^{\mathrm{gl}}\right)^{-1} \text { and } \beta=-2 \gamma^{\mathrm{gh}}-\gamma^{\mathrm{gl}},
$$

where $\gamma^{\mathrm{gh}}$ and $\gamma^{\mathrm{gl}}$ stand for the ghost and gluon wave function ADs respectively [31, 32].

Let us consider the following combination of the ghost and gluon self-energies

$$
F\left(a, \ell_{\mu}\right)=\left(1+\Pi^{\mathrm{gh}}\right)^{2}\left(1+\Pi^{\mathrm{gl}}\right)=\left(Z_{a}\right)^{-1}\left(1+\Pi_{B}^{\mathrm{gh}}\right)^{2}\left(1+\Pi_{B}^{\mathrm{gl}}\right) .
$$

This is a p-function with the evolution equation

$$
\left(\frac{\partial}{\partial L}+\beta a \frac{\partial}{\partial a}\right) F=\beta F \text {. }
$$

The $\beta$-function coefficient $\beta_{3}$ is a rational number while $\beta_{4}$ does depend on $\zeta_{3}$. This is the only specific information on the $\beta$-function which is needed for our analysis of the transcendental structure of $F_{4}$ and the coefficients $\beta_{4}$ and $\beta_{5}$ below. Indeed, the following is true:

Lemma. If $F$ is defined by eq. (5.2) and $\beta_{3}^{\zeta_{3}}=\beta_{4}^{\zeta_{5}}=0$ then

1. The coefficients $g_{i} \stackrel{\pi}{=} 0$ if $i \leq 3$.

2. The coefficient $\beta_{4} \stackrel{\pi}{=} 0$.

3. The coefficients $\left(Z_{a}\right)_{4, j}$ are rational for $j \geq 2$.

4. The coefficient $g_{4} \stackrel{\pi^{4}}{=} \frac{3}{8} \beta_{4}^{\zeta_{3}} \zeta_{4}$.

5. The coefficient $\beta_{5}^{\zeta_{6}}=0$.

The transcendental contributions to $\beta_{4}$ and $\beta_{5}$ are constrained by the following relation:

$$
\beta_{5}^{\zeta_{4}}=\frac{9}{8} \beta_{1} \beta_{4}^{\zeta_{3}} .
$$

\section{Proof.}

1. Indeed, the p-function $F_{3}$ is renormalized with the 3-loop RC $Z_{a}$ and, thus, can not depend on even zetas (see the discussion after (3.2) and section 7.1 of [1]).

2. This is a direct consequence of the fact that $\beta_{4} \equiv \gamma_{4}$ and $\gamma_{4} \stackrel{\pi}{=} 3 \beta_{1} g_{3}$.

3. This is an obvious consequence of the well-known fact that higher poles in a $\mathrm{RC} Z$ at loop order $(L+1)$ are completely fixed by simple pole terms of $Z$ and $Z_{a}$ with loop order not exceeding $L$ (so-called 't Hooft's constraints [22]). The constraints read

$$
a \frac{\partial Z_{*, k+1}}{\partial a}=\left[\beta a \frac{\partial}{\partial a}-\gamma\right] Z_{*, k},
$$

with $Z_{*, k}=\sum_{i \geq k} Z_{i k} a^{i}$. 
4. The renormalized function $F_{4}$ can be presented as:

$$
F_{4}=-\left(Z_{a}\right)_{4,1}^{\zeta_{3}} \zeta_{3} \frac{a^{4}}{\epsilon}+\sum_{i} z_{i}(\epsilon) p_{i} a^{4} \quad+\text { terms of order } a^{3} \text { and lower } .
$$

Literally repeating considerations after eq. (3.5) we arrive at:

$$
g_{4} \stackrel{\pi}{=}\left(Z_{a}\right)_{4,1}^{\zeta_{3}} \frac{3}{2} \zeta_{4} \equiv \frac{3}{8} \beta_{4}^{\zeta_{3}} \zeta_{4} .
$$

5. Now we consider the p-function $F_{5 B}^{\mathrm{si}}$. By construction, its renormalized version can be written as

$$
F_{5}^{\mathrm{si}}\left(a, \ell_{\mu}\right)=F_{5, B}^{\mathrm{si}}\left(a_{B}, \ell_{\mu}\right), \quad a_{B}=\mu^{2 \epsilon}\left(Z_{a}\right)_{4} a,
$$

where we have explicitly indicated that higher than 4-loop contributions to RC $Z_{a}$ have no effect on renormalization of $F_{5, B}^{\mathrm{si}}$ (as the latter starts from $\beta_{1} a_{B}$ ). As a result we have a representation

$$
F_{5}^{\mathrm{si}}=\left(Z_{a}\right)_{4,1}^{\zeta_{3}} \zeta_{3} \frac{a^{5}}{\epsilon} \beta_{1}+\sum_{i} z_{i}(\epsilon) p_{i} a^{5} \quad+\text { terms of order } a^{4} \text { and lower }
$$

where all $z_{i}$ are polynomial in $1 / \epsilon$ with rational coefficients and $p_{i}$ are some p-integrals with loop number less or equal 5. Assuming that all p-integrals in (5.9) are rewritten in term of hatted zetas and requiring finiteness of $F_{5}^{\text {si }}$ at $\epsilon \rightarrow 0$ we immediately obtain:

$$
d_{4} \stackrel{\pi}{=}-\frac{3}{8} \beta_{1} \beta_{4}^{\zeta_{3}} \zeta_{4},
$$

where we have used eq. (3.1) and the identity $\left(Z_{a}\right)_{4,1}=\frac{1}{4} \beta_{4}$. On the other hand, from (2.20) and first two statements of the Lemma we have

$$
d_{4} \stackrel{\pi}{=} \beta_{5}-\frac{\beta_{4} \beta_{2}}{\beta_{1}}+\frac{4 \beta_{4} g_{1}}{3}-4 \beta_{1} g_{4} \stackrel{\pi}{=} \beta_{5}-4 \beta_{1} g_{4} .
$$

Finally, by combining eqs. (5.7), (5.10) and (5.11) we arrive at (5.4).

Thus, the puzzle discussed in the Introduction is solved: the surviving $\zeta_{4}$ in (generic) p-functions $\hat{F}_{5}^{\text {si }}$ and in $F_{5}^{\text {si }}$ are indeed removed by the $\zeta_{4}$ piece of the 5-loop coefficient $\beta_{5}$ which, however, is generated (and completely fixed) by $\beta_{1}$ and the $\zeta_{3}$ term in the 4-loop $\beta$-function.

\section{The $\pi-\zeta-\epsilon$ structure of p-integrals and renormalization constants}

It is instructive to look into the structure of the results obtained in [13, 25] for 11 4-loop QCD p-functions and corresponding 5-loop ADs as displayed by table 1. Is it possible to understand the regularities shown in table 1? Sure. 


\begin{tabular}{|c|c||c|c|}
\hline $\mathrm{L}$ & $\mathrm{p}$-integrals & $\mathrm{L}+1$ & $\mathrm{Z}$ \\
\hline 0 & rational & 1 & $\mathrm{rational} / \epsilon$ \\
1 & rational $/ \epsilon$ & 2 & $\mathrm{rational} / \epsilon^{2}$ \\
2 & $\zeta_{3}$ & 3 & $\zeta_{3} / \epsilon$ \\
3 & $\zeta_{3} / \epsilon, \zeta_{4}, \zeta_{5}$ & 4 & $\zeta_{3} / \epsilon^{2},\left\{\zeta_{4}, \zeta_{5}\right\} / \epsilon$ \\
4 & $\zeta_{3} / \epsilon^{2},\left\{\zeta_{4}, \zeta_{5}\right\} / \epsilon, \zeta_{3}^{2}, \zeta_{6}, \zeta_{7}$ & 5 & $\zeta_{3} / \epsilon^{3},\left\{\zeta_{4}, \zeta_{5}\right\} / \epsilon^{2},\left\{\zeta_{3}^{2}, \zeta_{6}, \zeta_{7},\right\} / \epsilon$ \\
\hline
\end{tabular}

Table 1. The structure of p-integrals (expanded in $\epsilon$ up to and including the constant $\epsilon^{0}$ part) and $\mathrm{RCs}$ in dependence on the loop number $L$. The inverse power of $\epsilon$ stands for the maximal one in generic case; in particular cases it might be less.

1. The rationality ${ }^{7}$ of any 1-loop p-function is essentially made by hand by the choice of the G-scheme. 1-loop RC are, in fact, rational in any minimal scheme be it MS-, $\overline{\mathrm{MS}}$ - or G-scheme. The irrational structure of the p-functions at 2,3 and 4 loop level directly follows from explicit results for the corresponding master integrals as found in [1].

The fact that the set of zetas appearing in $(L+1)$ RCs is the same as the one displayed by $L$-loop $p$-integrals directly follows from the following theorem proven in [33].

Theorem 1. Any (L+1)-loop UV counterterm for any Feynman integral may be expressed in terms of pole and finite parts of some appropriately constructed L-loop p-integrals.

The maximal power of $1 / \epsilon$ with which a given zeta may appear in a $L$-loop contribution to a renormalization constant $\mathrm{Z}$ directly comes from the 't Hooft constraint (5.5).

Note that points 1. and 2. are also covered by remarks 1 . and 2. on page 13 of [34].

2. An attentive reader has probably noticed that table 1 does not include a particular combination of the transcendental weight 7 , namely, $\zeta_{3} \zeta_{4}$. There is a nice explanation of the fact. Indeed, let us take an arbitrary 4-loop p-integral $F$. If we consider it as a function of the irrational constants $\zeta_{3}, \ldots, \zeta_{7}$, then the following identity holds (see eq. (3.1))

$$
F\left(\zeta_{3}, \zeta_{4}, \zeta_{5}, \zeta_{6}, \zeta_{7}\right)=F\left(\hat{\zeta}_{3}, 0, \hat{\zeta}_{5}, 0, \hat{\zeta}_{7}\right)+\mathcal{O}(\epsilon)
$$

If a particular combination $\zeta_{3} \zeta_{4}$ could appear in the finite part of the function

$$
F\left(\zeta_{3}, \zeta_{4}, \zeta_{5}, \zeta_{6}, \zeta_{7}\right)
$$

then its hatted version would, obviously, contain a term proportional to ${\hat{\zeta_{3}}}^{2} / \epsilon$. This would imply that the constant part of a 3-loop p-integral contained $\zeta_{3}^{2}$ which is in contradiction to the previous point.

\footnotetext{
${ }^{7}$ In the sense of absence any irrational constants.
} 


\section{$7 \pi$-free representation for 5 - and 6-loop p-integrals}

In sections 3,4 , and 5 we have assumed that all encountered p-integrals are $\pi$-safe. In fact, for the treatment of these 3 sections to be valid the proven existence of $\pi$-free form for 4-loop p-integrals is just enough. Indeed, all currently known $p$-functions $F_{4}$ can be separated into 2 classes.

I. The lowest order contribution - the 1 in (2.1) - corresponds to a tree-level (that is loopless) diagram. The class includes QCD propagators and vertex functions (the latter in the propagator-like kinematical regime). Here the only contributions to the scale-invariant p-function $F_{5}^{\text {si }}$ with loop number equal to 5 could come from the derivative $\frac{\partial}{\partial \ell_{\mu}} F_{5}$. This derivative annihilates the constant part of the corresponding 5-loop p-integrals and, thus, does depend only on their pole parts.

The Adler function in 5-loops also belongs to class I in spite of the fact that its lowest order term corresponds to a 1-loop diagram. This is because by construction (i) the function is scale-invariant and (ii) it is completely determined by the non-constant part of the polarization operator.

Thus, the no- $\pi$ theorem for class I requires the existence of the $\pi$-free form only for the pole part of an arbitrary 5-loop p-integral (Property I).

II. Any correlator $\Pi\left(a, Q^{2}\right)$ of 2 composite operators with non-zero ADs. Here the starting object $F\left(a, Q^{2}\right)$ is constructed by a repeated application of the operator $\frac{\partial}{\partial Q^{2}}$ to $\Pi$ in order to arrive to the superficially convergent p-function $F$. During this procedure the bare pintegrals are effectively multiplied by $\epsilon$. The lowest order contribution to $F$ starts (at least) from the one-loop term. Current technology allow to compute $F_{4}$ and the corresponding $\mathrm{AD},{ }^{8} \gamma$, up to and including 5 loops. As a result the p-function $F_{5}^{s i}$ is contributed by 6-loop diagrams! However, a little meditation shows that only $1 / \epsilon^{2}$ and higher poles in these integrals could really contribute to $F_{5}^{s i}$.

Thus, the no- $\pi$ theorem for class II requires the existence of the $\pi$-free form only for arbitrary 6-loop p-integrals multiplied by $\epsilon^{2}$ (Property II).

Both Properties I and II are direct consequences of the following

Theorem 2. Let us assume that a $\pi$-free form exists for all L-loop p-integrals. Then

1. any $(L+1)$-loop p-integral times $\epsilon$ is $\pi$-safe.

2. any $(L+2)$-loop p-integral times $\epsilon^{2}$ is $\pi$-safe.

\section{Proof.}

1. Let us define the $\widehat{\mathrm{G}}$-scheme by pretending that hatted zetas do not depend on $\epsilon$. This means that all p-integrals are assumed to be expressed in term of the hatted zetas and that

\footnotetext{
${ }^{8}$ Which is, obviously, equal to the sum of the ADs of the both composite operators.
} 
the extraction of the pole part of a p-integral is defined as:

$$
\hat{K}\left(\mathcal{P}(\epsilon) \prod_{j} \hat{\zeta}_{j}\right):=\left(\sum_{i<0} \mathcal{P}_{i} \epsilon^{j}\right) \prod_{j} \hat{\zeta}_{j},
$$

with $\mathcal{P}(\epsilon)=\sum_{i} \epsilon^{i} \mathcal{P}_{i}$ being a polynomial in $\epsilon$ with rational coefficients. The corresponding coupling constant will be denoted as $\hat{a}$.

Theorem 1 insures then that all $3,4, \ldots,(L+1)$ counterterms are $\pi$-free ${ }^{9}$ in the new scheme. Let now $\langle\Gamma\rangle$ be an arbitrary $(L+1)$-loop p-integral (corresponding to a Feynman graph $\Gamma$ ). Its $\widehat{\mathrm{G}}$-renormalized (and, consequently, finite at $\epsilon \rightarrow 0$ ) version reads

$$
R\langle\Gamma\rangle\left(Q^{2}, \mu^{2}\right)=\langle\Gamma\rangle\left(Q^{2}, \mu^{2}\right)+Z_{\Gamma}+\sum_{\gamma} Z_{\gamma}\langle\Gamma / \gamma\rangle\left(Q^{2}\right)+\ldots
$$

Here $Z_{\gamma}$ is the UV Z-factor corresponding to a OPI subgraph $\gamma$ of $\Gamma, Z_{\Gamma}$ is the UV counterterm for the very Feynman integral $\langle\Gamma\rangle$ and dots stand for contributions with two and more UV subtractions. The finiteness of $R\langle\Gamma\rangle$ implies that

$$
\epsilon\langle\Gamma\rangle\left(Q^{2}, \mu^{2}\right)=-\epsilon\left(Z_{\Gamma}+\sum_{\gamma} Z_{\gamma}\langle\Gamma / \gamma\rangle\left(Q^{2}\right)+\ldots\right)+\mathcal{O}(\epsilon) .
$$

Note that the r.h.s. of eq. (7.2) includes only bare p-integrals with loop number not exceeding $L$ as well as $\pi$-free UV counterterms. Point 1 . is proven.

2. Let us consider (7.2) with $\langle\Gamma\rangle$ being an $(L+2)$-loop p-integral. Every particular term in the boxed part of eq. (7.2) is a product of some ( $\mu$-independent!) Z-factors and a reduced Feynman integral, the latter by construction includes a factor $\left(\mu^{2}\right)^{n \epsilon}$, with $n$ being its loop number. The very $p$-integral $\langle\Gamma\rangle\left(Q^{2}, \mu^{2}\right)$ depends on $\mu$ via a factor $\left(\mu^{2}\right)^{(L+2) \epsilon}$. Thus, after applying the operator $\mu^{2} \frac{\partial}{\partial \mu^{2}}$ to eq. (7.3) we obtain

$$
\epsilon^{2}\langle\Gamma\rangle\left(Q^{2}, \mu^{2}\right)=-\epsilon^{2}\left(\sum_{\gamma} Z_{\gamma}\left(1-\frac{L(\gamma)}{L+2}\right)\langle\Gamma / \gamma\rangle\left(Q^{2}\right)+\ldots\right)+\mathcal{O}(\epsilon),
$$

where $L(\gamma)$ is the loop number of the subgraph $\gamma$. Let us demonstrate that eq. (7.4) indeed proves point 2. First, in the $\widehat{\mathrm{G}}$-scheme every $\mathrm{RC} Z_{\gamma}$ in its r.h.s. is $\pi$-free as its loop number can not exceed $(L+1)$. Second, one can also check that every term $Z_{\gamma} \epsilon^{2}\langle\Gamma / \gamma\rangle$ is $\pi$-safe p-integral. For instance, if $L(\gamma)=1$ then $\langle\Gamma / \gamma\rangle$ is an $(L+1)$-loop p-integral and $Z_{\gamma}=C / \epsilon$, with $C$ being an $\epsilon$-independent constant. As a result we find that

$$
Z_{\gamma} \epsilon^{2}\langle\Gamma / \gamma\rangle=C \epsilon\langle\Gamma / \gamma\rangle
$$

is a $\pi$-safe combination due to point 1 .

\footnotetext{
${ }^{9}$ We always assume that the dependence of hatted zetas on normal ones is polynomial in $\epsilon$.
} 


\section{Adler function at order $\alpha_{s}^{5}$}

It has been conjectured in [2] that " $a \zeta_{4}$ term should arise in the Adler function at order $\alpha_{s}^{5}$ in the $\overline{\mathrm{MS}}$-scheme, and that this term is expected to disappear in the C-scheme as well".

Our analysis certainly confirms the conjecture and upgrade it to a firm prediction provided that the product $\epsilon F\left(Q^{2}\right)$ is $\pi$-safe for every 6-loop p-integral $F\left(Q^{2}\right)$. Due to Theorem 2 the condition is equivalent to the $\pi$-safeness of the whole class of 5 -loop pintegrals. Fortunately, very recently the last statement has received strong support from explicit evaluation of many 5-loop master p-integrals in [34].

\section{All order no- $\pi$ theorem}

In this and next sections we will consider the case of a generic one-charge theory, not necessarily QCD. We will not assume any specific properties of the corresponding $\beta$ function and $\mathrm{ADs}$, in particular $\beta_{3}^{\zeta_{3}}$ can have non-zero value.

In fact, the $\hat{G}$-scheme suggests a strong generalization of the no- $\pi$ theorem proven in section 3. Indeed, the following statement is true.

All order no- $\pi$ theorem. Let $F$ be any $L$-loop massless correlator and all $L$-loop pintegrals form a $\pi$-safe class. Then $F$ is $\pi$-free in any (massless) renormalization scheme for which corresponding $\beta$-function and $\mathrm{AD} \gamma$ are both $\pi$-free at least at the level of $L+1$ loops.

Proof. The theorem is obviously true for the $\hat{G}$-scheme. Let us denote the corresponding coupling constant as $\hat{a},(\pi$-free!) RG functions as $\hat{\beta}(\hat{a})$ and $\hat{\gamma}(\hat{a})$, and the very function $F$ as $\hat{F}$. For a different scheme the functions $F(a), \beta(a)$ and $\gamma(a)$ are related via the standard conversion formulas:

$$
\begin{aligned}
a & =\hat{a}\left(1+\sum_{1 \leq i \leq L} \hat{c}_{i} \hat{a}^{i}\right), \\
F(a) & =\left(1+\sum_{1 \leq i \leq L} \hat{b}_{i} \hat{a}^{i}\right) \hat{F}(\hat{a}), \\
\beta(a) & =\hat{\beta}(\hat{a})+\left(\sum_{1 \leq i \leq L} i \hat{c}_{i} \hat{a}^{i}\right) /\left(1+\sum_{1 \leq i \leq L} \hat{c}_{i} \hat{a}^{i}\right), \\
\gamma(a) & =\hat{\gamma}(\hat{a})+\hat{\beta}(\hat{a})\left(\sum_{1 \leq i \leq L} i \hat{b}_{i} \hat{a}^{i}\right) /\left(1+\sum_{1 \leq i \leq L} \hat{b}_{i} \hat{a}^{i}\right),
\end{aligned}
$$

where its is understood that $\hat{a}$ in eqs. (9.2)-(9.4) is expressed in terms of $a$ with the use of the inverted version of (9.1).

As relation (9.1) connects two $\pi$-independent to order $(L+1)$ schemes all coefficients $c_{i}$ in it should be $\pi$-free (see the comment after eq. (2.15)).

Thus, $\hat{\beta}(\hat{a}(a))$ and $\hat{\gamma}(\hat{a}(a))$ are $\pi$-free to order $(L+1)$. Finally, the requirement that $\gamma(a) \stackrel{\pi}{=} \mathcal{O}\left(a^{L+2}\right)$ and eq. (9.4) mean all coefficients $b_{i}$ with $i=1, \ldots L$ should also be $\pi$-free. 


\section{Odd and even zetas in RG-functions up to 6 loops}

The $\hat{G}$-scheme has some remarkable features. Indeed, one can see just from its definition that the corresponding "hatted" Green function, ADs and $Z$-factors can be obtained from the normal (that is computed with the $G$-scheme) by very simple rules.

- As a first step we make a formal replacement of the coupling constant $a$ by $\hat{a}$ in every G-renormalized Green function, $\mathrm{AD}$ and Z-factor we want to transform to the $\hat{G}$ scheme.

- Renormalized Green function $\hat{F}(\hat{a})$ is obtained from $F(\hat{a})$ by setting to zero all even zetas in the latter (both are assumed as taken at $\epsilon=0$ ).

- The same rule works for ADs and $\beta$-functions.

- If $Z$ is a ( $G$-scheme) renormalization constant then one should not only nullify all even zetas in $Z(\hat{a})$ but also replace every odd zeta term in it with its "hatted" counterpart.

For future reference we write below the connection between hatted and normal zeta for the class of 5-loop p-integrals [34]:

$$
\begin{array}{ll}
\hat{\zeta}_{3}:=\zeta_{3}+\frac{3 \epsilon}{2} \zeta_{4}-\frac{5 \epsilon^{3}}{2} \zeta_{6}+\frac{21 \epsilon^{5}}{2} \zeta_{8}, & \hat{\zeta}_{5}:=\zeta_{5}+\frac{5 \epsilon}{2} \zeta_{6}-\frac{35 \epsilon^{3}}{4} \zeta_{8}, \\
\hat{\zeta}_{7}:=\zeta_{7}+\frac{7 \epsilon}{2} \zeta_{8}, & \hat{\varphi}:=\varphi-3 \epsilon \zeta_{4} \zeta_{5}+\frac{5 \epsilon}{2} \zeta_{3} \zeta_{6} \quad \text { and } \quad \hat{\zeta}_{9}:=\zeta_{9},
\end{array}
$$

where

$$
\varphi:=\frac{3}{5} \zeta_{5,3}+\zeta_{3} \zeta_{5}-\frac{29}{20} \zeta_{8}=\zeta_{6,2}-\zeta_{3,5} \approx-0.1868414
$$

and multiple zeta values are defined as ${ }^{10}$

$$
\zeta_{n_{1}, n_{2}}:=\sum_{i>j>0} \frac{1}{i^{n_{1}} j^{n_{2}}} .
$$

Once we know which objects appear at 5-loop p-integrals we can construct a second table displaying the structure of 5-loop p-integrals and 6-loop ADs (in constructing the table and in what follows we assume that all class of 5-loop p-integrals is $\pi$-safe [34]). Once again, some combinations including even zetas, namely, $\zeta_{4} \zeta_{5}$ and $\zeta_{3} \zeta_{6}$ do not appear in table 2 essentially due to the $\pi$-safeness of 5 -loop p-integrals as expressed by (10.1).

It is interesting to note that representation (10.1) is in a sense "too deep" for the task of presentation of 5-loop p-integrals in the hatted form. Indeed, a simple inspection of table 2 clearly shows that the hatted form of a 5-loop p-integral can not depend on the last available terms in $\epsilon$-expansions of $\hat{\zeta}_{3}, \hat{\zeta}_{5}$ and $\hat{\varphi}$ (we do not consider the higher than $\epsilon^{0}$ terms in the $\epsilon$-expansion of 5 -loop p-integrals).

\footnotetext{
${ }^{10}$ Note, that the papers $[5,34]$ use somewhat different notation for multiple zeta values: $\left[\zeta_{n_{1}, n_{2}}\right]^{\text {our }}=$ $\left[\zeta_{n_{2}, n_{1}}\right]^{[5,34]}$. The definition (10.3) is in agreement to the one employed, e.g. in $[4,35-37]$.
} 


\begin{tabular}{|c|c||c|c|}
\hline $\mathrm{L}$ & $\mathrm{p}$-integrals & $\mathrm{L}+1$ & $\mathrm{Z}$ \\
\hline 5 & $\zeta_{3} / \epsilon^{3},\left\{\zeta_{4}, \zeta_{5}\right\} / \epsilon^{2},\left\{\zeta_{3}^{2}, \zeta_{6}, \zeta_{7}\right\} / \epsilon$, & 6 & $\zeta_{3} / \epsilon^{4},\left\{\zeta_{4}, \zeta_{5}\right\} / \epsilon^{3},\left\{\zeta_{3}^{2}, \zeta_{6}, \zeta_{7}\right\} / \epsilon^{2}$, \\
& $\zeta_{3} \zeta_{4}, \zeta_{8}, \zeta_{3} \zeta_{5}, \varphi, \zeta_{3}^{3}, \zeta_{9}$ & & $\left\{\zeta_{3} \zeta_{4}, \zeta_{8}, \zeta_{3} \zeta_{5}, \varphi, \zeta_{3}^{3}, \zeta_{9}\right\} / \epsilon$ \\
\hline
\end{tabular}

Table 2. The structure of 5-loop p-integrals (expanded in $\epsilon$ up to and including the constant $\epsilon^{0}$ part) and 6-loop RCs. The inverse power of $\epsilon$ stands for the maximal one in generic case; in particular cases it might be less.

Our next aim is to find explicitly the coefficients $c_{i}$ in the relation between the charge RCs $\hat{Z}_{a}$ and $Z_{a}$ in $\hat{G}$ - and $G$-schemes

$$
\hat{a}=a\left(1+\sum_{1 \leq i \leq L} c_{i} a^{i}\right) .
$$

As the bare charge must not depend on the choice of the renormalization scheme the coefficients $c_{i}$ are fixed by requiring that

$$
Z_{a} a=\hat{Z}_{a}(\hat{a}) \hat{a},
$$

where $\hat{a}$ is expressed in terms of $a$ via eq. (10.4). A simple counting of powers of $a$ in (10.5) shows that one can find the coefficients $c_{1} \ldots c_{6}$ in terms of $\beta_{1} \ldots \beta_{6}$.

For simplicity we start from the case of 4 loops. On general grounds we can write

$$
\beta=\beta_{1} a+\beta_{2} a^{2}+\left(r_{3}+\beta_{3}^{\zeta_{3}} \zeta_{3}\right) a^{3}+\left(r_{4}+\beta_{4}^{\zeta_{3}} \zeta_{3}+\beta_{4}^{\zeta_{4}} \zeta_{4}+\beta_{4}^{\zeta_{5}} \zeta_{5}\right) a^{4},
$$

where $r_{i}$ is $\beta_{i}$ with all zetas set to zero. The corresponding RCs $Z_{a}$ and $\hat{Z}_{a}$ read:

$$
\begin{aligned}
Z_{a}= & 1+\frac{a \beta_{1}}{\epsilon}+a^{2}\left(\frac{1}{2 \epsilon} \beta_{2}+\frac{1}{\epsilon^{2}} \beta_{1}^{2}\right)+a^{3}\left(\frac{1}{3 \epsilon}\left(r_{3}+\beta_{3}^{\zeta_{3}} \zeta_{3}\right)+\frac{7}{6 \epsilon^{2}} \beta_{1} \beta_{2}+\frac{1}{\epsilon^{3}} \beta_{1}^{3}\right) \\
& +a^{4}\left(\frac{1}{4 \epsilon}\left(r_{4}+\beta_{4}^{\zeta_{3}} \zeta_{3}+\beta_{4}^{\zeta_{4}} \zeta_{4}+\beta_{4}^{\zeta_{5}} \zeta_{5}\right)+\frac{1}{\epsilon^{2}}\left(\frac{5}{6} \beta_{1} r_{3}+\frac{5}{6} \beta_{1} \beta_{3}^{\zeta_{3}} \zeta_{3}+\frac{3}{8} \beta_{2}^{2}\right)\right. \\
& \left.+\frac{23}{12 \epsilon^{3}} \beta_{1}^{2} \beta_{2}+\frac{1}{\epsilon^{4}} \beta_{1}^{4}\right)
\end{aligned}
$$

and

$$
\begin{aligned}
\hat{Z}_{a}= & 1+\frac{\hat{a}}{\epsilon} \beta_{1}+\hat{a}^{2}\left(\frac{1}{2 \epsilon} \beta_{2}+\frac{1}{\epsilon^{2}} \beta_{1}^{2}\right)+\hat{a}^{3}\left(\frac{1}{3 \epsilon}\left(r_{3}+\beta_{3}^{\zeta_{3}} \hat{\zeta}_{3}\right)+\frac{7}{6 \epsilon^{2}} \beta_{1} \beta_{2}+\frac{1}{\epsilon^{3}} \beta_{1}^{3}\right) \\
& +\hat{a}^{4}\left(\frac{1}{4 \epsilon}\left(r_{4}+\beta_{4}^{\zeta_{3}} \hat{\zeta}_{3}+\beta_{4}^{\zeta_{5}} \hat{\zeta}_{5}\right)+\frac{1}{\epsilon^{2}}\left(\frac{5}{6} \beta_{1} r_{3}+\frac{5}{6} \beta_{1} \beta_{3}^{\zeta_{3}} \hat{\zeta}_{3}+\frac{3}{8} \beta_{2}^{2}\right)\right. \\
& \left.+\frac{23}{12 \epsilon^{3}} \beta_{1}^{2} \beta_{2}+\frac{1}{\epsilon^{4}} \beta_{1}^{4}\right) .
\end{aligned}
$$

Equation (10.5) can be easily solved with the result

$$
\begin{aligned}
c_{1}= & c_{2}=0, \\
c_{3}= & -\frac{1}{2} \beta_{3}^{\zeta_{3}} \zeta_{4}+\frac{5 \epsilon^{2}}{6} \beta_{3}^{\zeta_{3}} \zeta_{6}-\frac{7 \epsilon^{4}}{2} \beta_{3}^{\zeta_{3}} \zeta_{8}, \\
c_{4}= & \frac{1}{4 \epsilon}\left(\beta_{4}^{\zeta_{4}}-\beta_{1} \beta_{3}^{\zeta_{3}}\right) \zeta_{4}-\frac{3}{8} \beta_{4}^{\zeta_{3}} \zeta_{4}-\frac{5}{8} \beta_{4}^{\zeta_{5}} \zeta_{6} \\
& +\frac{5 \epsilon}{12} \beta_{1} \beta_{3}^{\zeta_{3}} \zeta_{6}+\epsilon^{2}\left(\frac{5}{8} \beta_{4}^{\zeta_{3}} \zeta_{6}+\frac{35}{16} \beta_{4}^{\zeta_{5}} \zeta_{8}\right)-\frac{7 \epsilon^{3}}{4} \beta_{1} \beta_{3}^{\zeta_{3}} \zeta_{8}-\frac{21 \epsilon^{4}}{8} \beta_{4}^{\zeta_{3}} \zeta_{8} .
\end{aligned}
$$


As the coefficients $c_{i}$ have to be finite at $\epsilon \rightarrow 0$ we arrive at the connection

$$
\beta_{4}^{\zeta_{4}}=\beta_{1} \beta_{3}^{\zeta_{3}}
$$

which has been already established in section 3 (see eq. (3.10)). Repeating the same reasonings for 5 and 6 loops we arrive at exact relations which express all $\pi$-dependent terms in the coefficients $\beta_{5}$ and $\beta_{6}$ in terms of $\beta_{1}, \beta_{2}$ and contributions (proportional to odd zetas) to $\beta_{3}, \beta_{4}$ and $\beta_{3}, \beta_{4}, \beta_{5}$ correspondingly. The relations read

$$
\begin{aligned}
\beta_{5}^{\zeta_{4}} & =\frac{1}{2} \beta_{2} \beta_{3}^{\zeta_{3}}+\frac{9}{8} \beta_{1} \beta_{4}^{\zeta_{3}}, \\
\beta_{5}^{\zeta_{6}} & =\frac{15}{8} \beta_{1} \beta_{4}^{\zeta_{5}}, \\
\beta_{5}^{\zeta_{3} \zeta_{4}} & =0 \\
\beta_{6}^{\zeta_{4}} & =\frac{3}{4} \beta_{2} \beta_{4}^{\zeta_{3}}+\frac{6}{5} \beta_{1} \beta_{5}^{\zeta_{3}}, \\
\beta_{6}^{\zeta_{6}} & =-\beta_{1}^{3} \beta_{3}^{\zeta_{3}}+\frac{5}{4} \beta_{2} \beta_{4}^{\zeta_{5}}+2 \beta_{1} \beta_{5}^{\zeta_{5}}, \\
\beta_{6}^{\zeta_{8}} & =\frac{14}{5} \beta_{1} \beta_{5}^{\zeta_{7}}, \\
\beta_{6}^{\zeta_{3} \zeta_{4}} & =\frac{12}{5} \beta_{1} \beta_{5}^{\zeta_{3}^{2}}, \\
\beta_{6}^{\zeta_{3} \zeta_{6}} & =0, \\
\beta_{6}^{\zeta_{4} \zeta_{5}} & =0 .
\end{aligned}
$$

In exactly the same way we derive similar constraints on a generic $\mathrm{AD} \gamma$. Indeed, let $F_{L}\left(a, \ell_{\mu}\right)$ is a p-function with evolution eq. (2.4) and $\hat{F}_{L}\left(\hat{a}, \ell_{\mu}\right)$ is its $\hat{G}$-scheme counterpart. Both functions should be finite at $\epsilon \rightarrow 0$ and meet a conversion relation:

$$
\hat{F}=\left(1+\sum_{1 \leq i \leq L} b_{i} a^{i}\right) F+\mathcal{O}\left(a^{L+1}\right)
$$

or, equivalently,

$$
\hat{Z}(\hat{a})=\left(1+\sum_{1 \leq i \leq L} b_{i} a^{i}\right) Z(a)+\mathcal{O}\left(a^{L+1}\right) .
$$

The finiteness of the coefficients $b_{i}$ leads to the following constraints

$$
\begin{aligned}
\gamma_{4}^{\zeta_{4}} & =\frac{3}{2} \gamma_{3}^{\zeta_{3}} \beta_{1}-\frac{1}{2} \gamma_{1} \beta_{3}^{\zeta_{3}}, \\
\gamma_{5}^{\zeta_{4}} & =\frac{3}{2} \gamma_{4}^{\zeta_{3}} \beta_{1}+\frac{3}{2} \gamma_{3}^{\zeta_{3}} \beta_{2}-\gamma_{2} \beta_{3}^{\zeta_{3}}-\frac{3}{8} \gamma_{1} \beta_{4}^{\zeta_{3}}, \\
\gamma_{5}^{\zeta_{6}} & =\frac{5}{2} \gamma_{4}^{\zeta_{5}} \beta_{1}-\frac{5}{8} \gamma_{1} \beta_{4}^{\zeta_{5}}, \\
\gamma_{5}^{\zeta_{3} \zeta_{4}} & =0
\end{aligned}
$$




$$
\begin{aligned}
\gamma_{6}^{\zeta_{4}} & =\frac{3}{2} \gamma_{5}^{\zeta_{3}} \beta_{1}+\frac{3}{2} \gamma_{4}^{\zeta_{3}} \beta_{2}+\frac{3}{2} \gamma_{3}^{\zeta_{3}} r_{3}-\frac{3}{2}\left(\gamma_{3}-\gamma_{3}^{\zeta_{3}} \zeta_{3}\right) \beta_{3}^{\zeta_{3}}-\frac{3}{4} \gamma_{2} \beta_{4}^{\zeta_{3}}-\frac{3}{10} \gamma_{1} \beta_{5}^{\zeta_{3}} \\
\gamma_{6}^{\zeta_{6}} & =\frac{5}{2} \gamma_{5}^{\zeta_{5}} \beta_{1}-\frac{5}{2} \gamma_{3}^{\zeta_{3}} \beta_{1}^{3}+\frac{5}{2} \gamma_{4}^{\zeta_{5}} \beta_{2}+\frac{3}{2} \gamma_{1} \beta_{1}^{2} \beta_{3}^{\zeta_{3}}-\frac{5}{4} \gamma_{2} \beta_{4}^{\zeta_{5}}-\frac{1}{2} \gamma_{1} \beta_{5}^{\zeta_{5}} \\
\gamma_{6}^{\zeta_{8}} & =\frac{7}{2} \gamma_{5}^{\zeta_{7}} \beta_{1}-\frac{7}{10} \gamma_{1} \beta_{5}^{\zeta_{7}} \\
\gamma_{6}^{\zeta_{3} \zeta_{4}} & =3 \gamma_{5}^{\zeta_{3}^{2}} \beta_{1}-\frac{3}{5} \gamma_{1} \beta_{5}^{\zeta_{3}^{2}} \\
\gamma_{6}^{\zeta_{3} \zeta_{6}} & =0 \\
\gamma_{6}^{\zeta_{4} \zeta_{5}} & =0
\end{aligned}
$$

Note, that if $\gamma=\beta$ then relations (10.15)-(10.17) become equivalent to (10.10)-(10.12) correspondingly.

We have successfully checked all the constraints above for a number of particular examples.

In QCD it was done for the 5-loop $\beta$-function and the quark mass $\mathrm{AD}$ known from $[8,16,17,38]$ as well as for all vertex and field ADs (taken in the Landau gauge) computed in [13].

At the level of 6 loops we have checked that the ( $\pi$-dependent contributions to) terms of order $n_{f}^{5} \alpha_{s}^{6}$ (for the $\beta$-functions) as well as terms of order $n_{f}^{5} \alpha_{s}^{6}$ and of order $n_{f}^{4} \alpha_{s}^{6}$ (the quark $\mathrm{AD})$ computed in [39-41] are in agreement with relations (10.12) and (10.17) respectively.

For the case of the normal QCD with the SU(3) gauge group our results for $\pi$-dependent contributions to the 6 -loop coefficients of the $\beta$-function and the quark mass $\mathrm{AD}$ read (the known terms are boxed)

$$
\begin{aligned}
\beta_{6} \stackrel{\pi}{=} & \frac{608}{405} n_{f}^{5} \zeta_{4}+n_{f}^{4}\left(\frac{164792}{1215} \zeta_{4}-\frac{1840}{27} \zeta_{6}\right)+n_{f}^{3}\left(-\frac{4173428}{405} \zeta_{4}+\frac{1800280}{243} \zeta_{6}\right) \\
& +n_{f}^{2}\left(\frac{68750632}{405} \zeta_{4}-\frac{13834700}{81} \zeta_{6}\right)+n_{f}\left(-\frac{146487538}{135} \zeta_{4}+\frac{40269130}{27} \zeta_{6}\right) \\
& +99\left(44213 \zeta_{4}-64020 \zeta_{6}\right), \\
\gamma_{6}^{m} \stackrel{\pi}{=} & \frac{320}{243} n_{f}^{5} \zeta_{4}+n_{f}^{4}\left(-\frac{90368}{405} \zeta_{4}+\frac{22400}{81} \zeta_{6}\right) \\
& +n_{f}^{3}\left(-\frac{92800}{27} \zeta_{3} \zeta_{4}-\frac{2872156}{405} \zeta_{4}+\frac{503360}{243} \zeta_{6}\right) \\
& +n_{f}^{2}\left(\frac{661760}{9} \zeta_{3} \zeta_{4}+\frac{155801234}{405} \zeta_{4}-\frac{378577520}{729} \zeta_{6}+\frac{12740000}{81} \zeta_{8}\right) \\
& +n_{f}\left(-\frac{1413280}{3} \zeta_{3} \zeta_{4}-\frac{4187656168}{1215} \zeta_{4}+\frac{5912758120}{729} \zeta_{6}-\frac{96071360}{27} \zeta_{8}\right) \\
& +3194400 \zeta_{3} \zeta_{4}+\frac{272688530}{81} \zeta_{4}-\frac{6778602160}{243} \zeta_{6}+15889720 \zeta_{8} .
\end{aligned}
$$

The results for generic gauge group are rather bulky, they can be found in Mathematica [42] and FORM [43] supplementary files attached to this paper. 
All 6-loop RG functions known from [3-5] for the case of the $O(n) \phi^{4}$ model agree with the constraints (provided that the transcendental constant $\zeta_{5,3}$ is replaced by $\varphi$ according to relation (10.2)).

\section{Odd and even zetas in p-functions up to 5 loops}

The knowledge of the coefficients $c_{i}$ and $b_{i}$ in the relations (10.4) and (10.13) allows to reconstruct the full p-function $F$ from its hatted version $\hat{F}$. Indeed, let

$$
F(a)=1+\sum_{1 \leq i \leq L} F_{i} a^{i} .
$$

Then

$$
\hat{F}(\hat{a})=1+\sum_{1 \leq i \leq L} \hat{F}_{i} \hat{a}^{i} \text { with } \hat{F}_{i}=\left.\left(F_{i}\right)\right|_{\pi=0} .
$$

At the limit of $\epsilon \rightarrow 0$ the coefficients $c_{i}$ and $b_{i}$ defined in the previous section read (the constraints (10.10)-(10.12) and (10.15)-(10.17) are taken into account; the results can be also found in computer-readable form attached to this paper as supplementary material)

$$
\begin{aligned}
c_{1} & =c_{2}=0, \\
c_{3} & =-\frac{1}{2} \beta_{3}^{\zeta_{3}} \zeta_{4}, \\
c_{4} & =-\frac{3}{8} \beta_{4}^{\zeta_{3}} \zeta_{4}-\frac{5}{8} \beta_{4}^{\zeta_{5}} \zeta_{6}, \\
c_{5} & =-\frac{3}{10} \beta_{5}^{\zeta_{3}} \zeta_{4}-\frac{3}{5} \beta_{5}^{\zeta_{3}^{2}} \zeta_{3} \zeta_{4}+\frac{1}{4} \beta_{1}^{2} \beta_{3}^{\zeta_{3}} \zeta_{6}-\frac{1}{2} \beta_{5}^{\zeta_{5}} \zeta_{6}-\frac{7}{10} \beta_{5}^{\zeta_{7}} \zeta_{8},
\end{aligned}
$$

and

$$
\begin{aligned}
& b_{1}=b_{2}=0, \\
& b_{3}=-\frac{1}{2} \gamma_{3}^{\zeta_{3}} \zeta_{4}, \\
& b_{4}=-\frac{3}{8} \gamma_{4}^{\zeta_{3}} \zeta_{4}-\frac{5}{8} \gamma_{4}^{\zeta_{5}} \zeta_{6}, \\
& b_{5}=-\frac{3}{10} \gamma_{5}^{\zeta_{3}} \zeta_{4}-\frac{3}{5} \gamma_{5}^{\zeta_{3}^{2}} \zeta_{4} \zeta_{3}+\left(-\frac{1}{2} \gamma_{5}^{\zeta_{5}}+\frac{1}{2} \beta_{1}^{2} \gamma_{3}^{\zeta_{3}}-\frac{1}{4} \beta_{3}^{\zeta_{3}} \beta_{1} \gamma_{1}\right) \zeta_{6}-\frac{7}{10} \gamma_{5}^{\zeta_{7}} \zeta_{8} .
\end{aligned}
$$

Finally, we can now restore all $\pi$-dependent terms in $F_{1}-F_{5}$ from eqs. (10.13), (11.3) and (11.4) with the result:

$$
\begin{aligned}
F_{1}= & \hat{F}_{1}, \\
F_{2}= & \hat{F}_{2}, \\
F_{3}= & \hat{F}_{3}+\frac{1}{2} \gamma_{3}^{\zeta_{3}} \zeta_{4}, \\
F_{4}= & \hat{F}_{4}+\left(-\frac{1}{2} \hat{F}_{1} \beta_{3}^{\zeta_{3}}+\frac{3}{8} \gamma_{4}^{\zeta_{3}}+\frac{1}{2} \gamma_{3}^{\zeta_{3}} \hat{F}_{1}\right) \zeta_{4}+\frac{5}{8} \gamma_{4}^{\zeta_{5}} \zeta_{6}, \\
F_{5}= & \hat{F}_{5}+\left(-\beta_{3}^{\zeta_{3}} \hat{F}_{2}-\frac{3}{8} \hat{F}_{1} \beta_{4}^{\zeta_{3}}+\frac{3}{10} \gamma_{5}^{\zeta_{3}}+\frac{3}{8} \gamma_{4}^{\zeta_{3}} \hat{F}_{1}+\frac{1}{2} \gamma_{3}^{\zeta_{3}} \hat{F}_{2}\right) \zeta_{4}+\frac{3}{5} \gamma_{5}^{\zeta_{3}^{2}} \zeta_{4} \zeta_{3} \\
& +\left(\frac{1}{2} \gamma_{5}^{\zeta_{5}}+\frac{1}{4} \beta_{3}^{\zeta_{3}} \beta_{1} \gamma_{1}-\frac{5}{8} \hat{F}_{1} \beta_{4}^{\zeta_{5}}+\frac{5}{8} \hat{F}_{1} \gamma_{4}^{\zeta_{5}}-\frac{1}{2} \gamma_{3}^{\zeta_{3}} \beta_{1}^{2}\right) \zeta_{6}+\frac{7}{10} \gamma_{5}^{\zeta_{7}} \zeta_{8} .
\end{aligned}
$$


We have checked that eqs. (11.5)-(11.9) correctly describe the $\pi$-dependent contributions to all 11 4-loop p-functions from [25] as well as their 5-loop scale invariant versions constructed according to the definition (2.17) with the use of 5-loop ADs from [13].

\section{Discussion and conclusions}

We have clarified and proved the no- $\pi$ theorem for all one-scale RG-invariant Euclidean correlators $\hat{F}_{5}^{s i}$ first suggested in [2]. The theorem is extended to a case of generic Euclidean correlators with arbitrary high loop number.

We have found many new identities relating contributions proportional to odd and even zetas in generic $\overline{\mathrm{MS}} \mathrm{ADs}$ and $\beta$-functions. The new identities allow to reconstruct all $\pi$-dependent terms in a RG function at the level $L$ loops in terms of the same function and the $\beta$-function taken at loop level $(L-1)$ and less with $L=4,5$ and 6 . We have explicitly elaborated the corresponing predictions for $L=4,5$ and 6 and found full agreement of our results with calculated results where the latter are available.

For the case of the $O(n) \phi^{4}$ theory there exist since recently results for the corresponding $\mathrm{ADs}$ at the 7 loop level [4]. In fact, we have extended our formulas to this case and successfully reproduced all the $\pi$-dependent terms in 7-loop ADs and the 6-loop propagator of the scalar field. We are planning to publish a detailed account of these results in the near future.

Finally, the use of the $\widehat{\mathrm{G}}$-scheme is not limited to one-charge models or/and to the Landau gauge fixing. It is clear that the derivation of sections 10 and 11 can be straightforwardly extended on a general case of a QFT model with a few coupling constants considered in a generic covariant gauge. More loops are also not a problem provided corresponding generalizations of representation (10.1) can be constructed.

\section{Acknowledgments}

Both authors are indebted to J.H. Kühn for careful reading the manuscript and a lot of good advice.

We are very grateful to J. Gracey for his help in extracting 6-loop terms from generic results of [39-41] and to O. Schnetz for providing us with his results for the 7-loop ADs and the bare 6-loop propagator in the $O(n) \phi^{4}$ model.

The second author thanks J. Davies, M. Jamin, A. Kataev, B. Kniehl, M. Kompaniets, E. Panzer, A. Pikelner and A. Vogt for useful discussions.

The work of P.A. Baikov is supported in part by the grant RFBR 17-02-00175A of the Russian Foundation for Basic Research. The work by K.G. Chetykin was supported by the German Federal Ministry for Education and Research BMBF through Grant No. 05H15GUCC1.

Note added. After completing our work we have been informed by E. Panzer that he independently has found a way to reconstruct $\pi$-dependent terms in RG functions of the $O(n) \phi^{4}$ model. 
Open Access. This article is distributed under the terms of the Creative Commons Attribution License (CC-BY 4.0), which permits any use, distribution and reproduction in any medium, provided the original author(s) and source are credited.

\section{References}

[1] P.A. Baikov and K.G. Chetyrkin, Four Loop Massless Propagators: An Algebraic Evaluation of All Master Integrals, Nucl. Phys. B 837 (2010) 186 [arXiv:1004.1153] [INSPIRE].

[2] M. Jamin and R. Miravitllas, Absence of even-integer $\zeta$-function values in Euclidean physical quantities in QCD, Phys. Lett. B 779 (2018) 452 [arXiv:1711.00787] [INSPIRE].

[3] D.V. Batkovich, K.G. Chetyrkin and M.V. Kompaniets, Six loop analytical calculation of the field anomalous dimension and the critical exponent $\eta$ in $O(n)$-symmetric $\varphi^{4}$ model, Nucl. Phys. B 906 (2016) 147 [arXiv:1601.01960] [INSPIRE].

[4] O. Schnetz, Numbers and Functions in Quantum Field Theory, Phys. Rev. D 97 (2018) 085018 [arXiv:1606.08598] [INSPIRE].

[5] M.V. Kompaniets and E. Panzer, Minimally subtracted six loop renormalization of $O(n)$-symmetric $\phi^{4}$ theory and critical exponents, Phys. Rev. D 96 (2017) 036016 [arXiv: 1705. 06483] [INSPIRE].

[6] S.G. Gorishnii, A.L. Kataev and S.A. Larin, The $O\left(\alpha_{s}^{3}\right)$-corrections to $\sigma_{\text {tot }}\left(e^{+} e^{-} \rightarrow\right.$ hadrons $)$ and $\Gamma\left(\tau^{-} \rightarrow \nu_{\tau}+\right.$ hadrons) in QCD, Phys. Lett. B 259 (1991) 144 [INSPIRE].

[7] P.A. Baikov, K.G. Chetyrkin and J.H. Kühn, Adler Function, Bjorken Sum Rule and the Crewther Relation to Order $\alpha_{s}^{4}$ in a General Gauge Theory, Phys. Rev. Lett. 104 (2010) 132004 [arXiv:1001.3606] [INSPIRE].

[8] P.A. Baikov, K.G. Chetyrkin and J.H. Kühn, Five-loop fermion anomalous dimension for a general gauge group from four-loop massless propagators, JHEP 04 (2017) 119 [arXiv: 1702.01458] [INSPIRE].

[9] D. Boito, M. Jamin and R. Miravitllas, Scheme Variations of the QCD Coupling and Hadronic $\tau$ Decays, Phys. Rev. Lett. 117 (2016) 152001 [arXiv:1606.06175] [INSPIRE].

[10] P.A. Baikov, K.G. Chetyrkin and J.H. Kühn, Scalar correlator at $\mathcal{O}\left(\alpha_{s}^{4}\right)$, Higgs decay into b-quarks and bounds on the light quark masses, Phys. Rev. Lett. 96 (2006) 012003 [hep-ph/0511063] [INSPIRE].

[11] F. Herzog, B. Ruijl, T. Ueda, J.A.M. Vermaseren and A. Vogt, On Higgs decays to hadrons and the $R$-ratio at $N^{4} L O, J H E P 08$ (2017) 113 [arXiv:1707.01044] [INSPIRE].

[12] J. Davies and A. Vogt, Absence of $\pi^{2}$ terms in physical anomalous dimensions in DIS: Verification and resulting predictions, Phys. Lett. B 776 (2018) 189 [arXiv:1711.05267] [INSPIRE].

[13] K.G. Chetyrkin, G. Falcioni, F. Herzog and J.A.M. Vermaseren, Five-loop renormalisation of QCD in covariant gauges, JHEP 10 (2017) 179 [arXiv:1709.08541] [INSPIRE].

[14] B. Ruijl, F. Herzog, T. Ueda, J.A.M. Vermaseren and A. Vogt, $R^{*}$-operation and five-loop calculations, in proceedings of 13 th International Symposium on Radiative Corrections: Application of Quantum Field Theory to Phenomenology (RADCOR2017), St. Gilgen, Austria, September 24-29, 2017 [arXiv: 1801.06084] [INSPIRE]. 
[15] P.A. Baikov, K.G. Chetyrkin and J.H. Kühn, Five-Loop Running of the QCD coupling constant, Phys. Rev. Lett. 118 (2017) 082002 [arXiv:1606.08659] [INSPIRE].

[16] F. Herzog, B. Ruijl, T. Ueda, J.A.M. Vermaseren and A. Vogt, The five-loop $\beta$-function of Yang-Mills theory with fermions, JHEP 02 (2017) 090 [arXiv:1701.01404] [INSPIRE].

[17] T. Luthe, A. Maier, P. Marquard and Y. Schröder, The five-loop $\beta$-function for a general gauge group and anomalous dimensions beyond Feynman gauge, JHEP 10 (2017) 166 [arXiv: 1709.07718] [INSPIRE].

[18] O.V. Tarasov, A.A. Vladimirov and A.Yu. Zharkov, The Gell-Mann-Low Function of QCD in the Three Loop Approximation, Phys. Lett. B 93 (1980) 429 [INSPIRE].

[19] S.A. Larin and J.A.M. Vermaseren, The Three loop QCD $\beta$-function and anomalous dimensions, Phys. Lett. B 303 (1993) 334 [hep-ph/9302208] [INSPIRE].

[20] T. van Ritbergen, J.A.M. Vermaseren and S.A. Larin, The Four loop $\beta$-function in quantum chromodynamics, Phys. Lett. B 400 (1997) 379 [hep-ph/9701390] [INSPIRE].

[21] M. Czakon, The Four-loop QCD $\beta$-function and anomalous dimensions, Nucl. Phys. B 710 (2005) 485 [hep-ph/0411261] [INSPIRE].

[22] G. 't Hooft, Dimensional regularization and the renormalization group, Nucl. Phys. B 61 (1973) 455 [inSPIRE].

[23] W.A. Bardeen, A.J. Buras, D.W. Duke and T. Muta, Deep Inelastic Scattering Beyond the Leading Order in Asymptotically Free Gauge Theories, Phys. Rev. D 18 (1978) 3998 [INSPIRE].

[24] K.G. Chetyrkin, A.L. Kataev and F.V. Tkachov, New Approach to Evaluation of Multiloop Feynman Integrals: The Gegenbauer Polynomial x Space Technique,

Nucl. Phys. B 174 (1980) 345 [InSPIRE].

[25] B. Ruijl, T. Ueda, J.A.M. Vermaseren and A. Vogt, Four-loop QCD propagators and vertices with one vanishing external momentum, JHEP 06 (2017) 040 [arXiv: 1703. 08532] [INSPIRE].

[26] P.A. Baikov, K.G. Chetyrkin and J.H. Kühn, Massless Propagators, R(s) and Multiloop QCD, Nucl. Part. Phys. Proc. 261-262 (2015) 3 [arXiv:1501.06739] [InSPIRE].

[27] R.N. Lee, A.V. Smirnov and V.A. Smirnov, Master Integrals for Four-Loop Massless Propagators up to Transcendentality Weight Twelve, Nucl. Phys. B 856 (2012) 95 [arXiv:1108.0732] [INSPIRE].

[28] J.A.M. Vermaseren, S.A. Larin and T. van Ritbergen, The four loop quark mass anomalous dimension and the invariant quark mass, Phys. Lett. B 405 (1997) 327 [hep-ph/9703284] [INSPIRE].

[29] J. Kubo, S. Sakakibara and P.M. Stevenson, The Renormalization Scheme Ambiguity and Perturbation Theory Near a Fixed Point, Phys. Rev. D 29 (1984) 1682 [InSPIRE].

[30] D.J. Broadhurst, Dimensionally continued multiloop gauge theory, hep-th/9909185 [INSPIRE].

[31] J.C. Taylor, Ward Identities and Charge Renormalization of the Yang-Mills Field, Nucl. Phys. B 33 (1971) 436 [inSPIRE].

[32] A. Blasi, O. Piguet and S.P. Sorella, Landau gauge and finiteness, Nucl. Phys. B 356 (1991) 154 [inSPIRE]. 
[33] K.G. Chetyrkin and V.A. Smirnov, R* Operation Corrected, Phys. Lett. B 144 (1984) 419 [INSPIRE].

[34] A. Georgoudis, V. Gonçalves, E. Panzer and R. Pereira, Five-loop massless propagator integrals, arXiv: 1802.00803 [INSPIRE].

[35] J. Blumlein, D.J. Broadhurst and J.A.M. Vermaseren, The Multiple Zeta Value Data Mine, Comput. Phys. Commun. 181 (2010) 582 [arXiv:0907.2557] [InSPIRE].

[36] D. Broadhurst, Multiple Zeta Values and Modular Forms in Quantum Field Theory, Springer Vienna, Vienna (2013) [INSPIRE].

[37] R.N. Lee and K.T. Mingulov, Introducing SummerTime: a package for high-precision computation of sums appearing in DRA method, Comput. Phys. Commun. 203 (2016) 255 [arXiv: 1507.04256] [INSPIRE].

[38] T. Luthe, A. Maier, P. Marquard and Y. Schröder, Five-loop quark mass and field anomalous dimensions for a general gauge group, JHEP 01 (2017) 081 [arXiv: 1612.05512] [INSPIRE].

[39] J.A. Gracey, The $Q C D \beta$-function at $\mathcal{O}\left(1 / N_{f}\right)$, Phys. Lett. B 373 (1996) 178 [hep-ph/9602214] [INSPIRE].

[40] M. Ciuchini, S.E. Derkachov, J.A. Gracey and A.N. Manashov, Quark mass anomalous dimension at $O\left(1 / N_{f}^{2}\right)$ in $Q C D$, Phys. Lett. B 458 (1999) 117 [hep-ph/9903410] [InSPIRE].

[41] M. Ciuchini, S.E. Derkachov, J.A. Gracey and A.N. Manashov, Computation of quark mass anomalous dimension at $O\left(1 / N_{f}^{2}\right)$ in quantum chromodynamics,

Nucl. Phys. B 579 (2000) 56 [hep-ph/9912221] [INSPIRE].

[42] Wolfram Research Inc., Mathematica, version 11.3.

[43] J.A.M. Vermaseren, New features of FORM, math-ph/0010025 [INSPIRE]. 